\title{
Heterogeneity in Preferences for Anti-coagulant Use in Atrial Fibrillation: A Latent Class Analysis
}

\author{
Janine van Til $^{1}(\mathbb{D}) \cdot$ Catharina Oudshoorn-Groothuis ${ }^{1}$ (D) Marieke Weernink $^{1,2}$ (D) Clemens von Birgelen $^{1,3}(\mathbb{D}$
}

Published online: 24 April 2020

(c) The Author(s) 2020

\begin{abstract}
Introduction Recent reviews on patients' preferences towards attributes of oral anti-coagulant therapy have shown that preference for convenience of therapy is heterogeneous. In this study, we used a novel approach-latent class analysis (LCA) - to assess heterogeneity.

Methods We developed a health preference survey that consisted of 12 discrete choice questions. The following attributes of convenience were included: intake frequency; need for regular coagulation monitoring; diet or drug interactions; relation between medication and food intake; and pill type. Background questions about gender, age, current therapy [i.e., directacting oral anti-coagulant (DOAC) or vitamin K antagonist (VKA)], self-reported medication adherence, and pill burden were included. Mixed logit analysis (MLA) and LCA were performed. The scale-adjusted LCA model with two scale classes and four preference classes emerged as the model with the best fit and interpretability.

Results A total of 508 patients with non-valvular atrial fibrillation from five European countries (Germany, Italy, Spain, France, and the UK) were surveyed in August 2017. The most important attributes were need for monitoring (37\%) and intake frequency (27\%). Patient preferences were significantly influenced by country, gender, and current anti-coagulant therapy. Four different preference classes of patients were identified in the LCA. First, most patients (57\%) were in the "no need for regular coagulation monitoring" class. Current DOAC users and patients who were the least adherent to therapy were more likely to prefer no coagulation monitoring. Second, $20 \%$ of patients were in the "balanced" class of patients. Current VKA users with moderate adherence were more likely to be in this class. Patients who reported the lowest adherence were most likely in the "once daily, interactions likely" class (16\%). Fourth, current VKA users and highly adherent patients were most likely to prefer therapies with a need for regular coagulation monitoring (7\%).

Conclusions This study demonstrated significant preference heterogeneity among patients with atrial fibrillation and linked these preferences to differences in background characteristics. Country of residence and currently prescribed therapy influenced patient preferences in both the MLA and LCA models.
\end{abstract}

Electronic supplementary material The online version of this article (https://doi.org/10.1007/s40271-020-00420-z) contains supplementary material, which is available to authorized users.

Janine van Til

j.a.vantil@utwente.nl

1 Department of Health Technology and Services Research, Technical Medical Centre, University of Twente, Enschede, The Netherlands

2 Municipal Health Services (GGD) Twente, Enschede, The Netherlands

3 Medical Spectrum Twente, Thorax Centrum Twente, Enschede, The Netherlands

\section{Introduction}

Non-valvular atrial fibrillation $(\mathrm{AF})$ is the most common heart rhythm disturbance [1]. AF is associated with a substantial increase in the risk of ischemic stroke [2]. Traditionally, vitamin K antagonists (VKAs) were prescribed to mitigate this risk. During the last decade, multiple directacting oral anti-coagulants (DOACs) have been approved for clinical use. This has broadened the options for anticoagulant therapy in patients with $\mathrm{AF}[3,4]$. Recent systematic reviews showed that DOACs are at least as effective as VKAs in preventing ischemic stroke and that some DOACs might have a better safety profile than VKAs $[3,4]$. Some 


\section{Key Points for Decision Makers}

The most important attributes of anti-coagulation therapy are the need for monitoring and intake frequency.

Preferences for anti-coagulation therapy in patients with atrial fibrillation differed between patients from the UK, Spain, Germany, France, and Italy, between men and woman, and between current users of vitamin K antagonists and direct-acting oral anti-coagulants.

Similar covariates that explained heterogeneity in preferences were identified with the latent class analysis and mixed logit analysis.

experts argue that DOACs should be the preferred choice because of their safety profile and the absence of a need for regular coagulation monitoring [5]. Others have stated that DOACs are associated with poor adherence and are not always superior to VKAs in routine clinical practice [6]. Yet, DOACs are regarded as a valuable alternative to VKAs, and their use is increasing [7].

The choice of therapy in AF is a preference-sensitive decision, determined by a trade-off between perceived advantages and disadvantages of VKAs and DOACs. In this context, European guidelines [7] advise consideration of patient-related clinical factors, product characteristics, and patient preference, thereby promoting shared decision making. Several recent studies addressed preference for anticoagulant therapy in patients with AF. To most patients, reducing stroke and bleeding risk is most important $[8,9]$, but most DOACs have similar benefit-risk profiles on a population level. DOACs differ mostly from VKAs in convenience attributes (e.g., intake frequency, regular coagulation monitoring, or drug/food interaction) [10]. With regard to convenience of treatment, once-daily intake and no need for regular coagulation monitoring were found to be the most important attributes of anti-coagulation therapy; however, there is considerable heterogeneity in patient preference $[8$, 9]. Heterogeneity in preference may be attributed to differences in sociodemographic characteristics, currently used anti-coagulation therapy, and preference elicitation method.

Previous studies used observational study designs, qualitative interviews, trade-off techniques such as standard gamble and time-trade off, conjoint analysis, the threshold technique, or discrete choice experiments to elicit preferences [8]. In this study, a discrete choice experiment was used to elicit preferences for therapy in $\mathrm{AF}$, because this technique enables us to estimate the impact of different attributes of therapy on preference for treatment, and different approaches to address heterogeneity in discrete choice experiment data, such as latent class analysis (LCA) and mixed logit analysis (MLA) are available [11].

The objective of this study was to further explore preference heterogeneity for convenience of anti-coagulant therapy in patients with AF. The second objective of the study was to use LCA, which has not been used in this context to study heterogeneity. The results of the LCA were compared with the results of an MLA.

The results should provide further insight for clinicians into the different trade-offs that they may expect in patients with $\mathrm{AF}$ who have to choose treatment. This knowledge may facilitate shared decision making, and it may ultimately help improve therapy adherence.

\section{Methods}

The good practice guidelines for conjoint analysis were used to develop the questionnaire that was used in this study [12, 13].

\subsection{Attributes of Convenience of Anti-coagulant Therapy}

The initial list of attributes of convenience in anti-coagulation therapy was determined by a combination of literature review, focus group sessions with patients in five European countries [10,14], and expert consultation. The initial list of attributes consisted of seven attributes: [1] need for coagulation monitoring; [2] dosing regimen; [3] intake frequency; [4] pill type; [5] interactions with foods and/or drugs; [6] need for bridging/antidote; and [7] distance to the practitioner. In an individual interview by phone, five experts in the treatment of patients with AF from the different countries in Europe were questioned about the convenience attributes they considered relevant in prescribing treatment. This did not result in additional attributes. In the second stage, the list of attributes from the literature was discussed. Based on this discussion, two attributes were omitted. Distance to the practitioner was irrelevant to this study since this attribute is not specific for patient or drug, but for the healthcare system. Bridging was omitted, because a trial demonstrated that not using bridging anti-coagulation was non-inferior to perioperative bridging [15]. The experts argued that patient education in clinical practice could take away fears about excessive bleeding as a result of anti-coagulation therapy in the case of accidents or surgery, and this should not be included as an argument for or against one type of anti-coagulant therapy. The final list of aspects of convenience consisted of five attributes with two or three levels: (1) intake frequency (once-daily/twice-daily); (2) regular coagulation monitoring and possible dose adjustments (required/not required); (3) diet and/or drug interactions (likely/unlikely); (4) pill 
intake (must be taken with food/can be taken with or without food); (5) pill type (capsule, tablet, or a tablet that dissolves in the mouth). The tablet that dissolves in the mouth is not a characteristic of a currently marketed product but was added as a potential improvement to existing therapy. In the survey, these attributes and their levels were explained to the respondents in lay language.

\subsection{Discrete Choice Experiment}

To elicit patient preferences, a discrete choice experiment was used, and each choice question consisted of two hypothetical anti-coagulation therapy scenarios. In each discrete choice question, patients were asked to indicate which anticoagulation therapy they would choose if the two therapies presented were the only two options available (Fig. 1). Prior to each question, patients were instructed that the safety and efficacy of both hypothetical therapies were equal. All possible combinations of attributes and levels resulted in a potential $48\left(2^{4} \times 3^{1}\right)$ scenarios and $2256(48 \times 47)$ choice sets. A fractional factorial design was developed in $\mathrm{R}$. The design consisted of 12 choice sets, randomly assigned over two versions of the survey. The design was fully balanced and orthogonal, with the three-level attribute appearing eight times and the two-level attribute appearing 12 times. No overlap was allowed.

\subsection{Questionnaire Design}

The discrete choice experiment was part of a larger questionnaire. This consisted of the following sections: (A) screening questions on age, gender, co-morbidity, and current and previous anti-coagulation therapy; (B) explanation of attributes and levels, after which patients were asked to select the characteristic (level) of their current therapy for each attribute; (C) discrete choice experiment regarding convenience of treatment, as described above; (D) a risk-benefit trade-off between stroke prevention and bleeding risk using a tradeoff exercise; (E) attitude and knowledge of anti-coagulation therapy [16]; and (F) general health and socio-demographic characteristics. The questionnaire was pre-tested in patients with and without $\mathrm{AF}$ to ensure comprehension and feasibility $(n=10)$.

\subsection{Data Collection}

Patients from five European countries (Germany, Italy, Spain, France, and the UK) taking oral anti-coagulants for AF-related stroke prevention were surveyed in August 2017. Patients were recruited on a voluntary basis via the Institute for Marketing Research (Frankfurt, Germany). This agency has a database of existing patients with $\mathrm{AF}$, and additional patients were invited to participate through their medical doctors. Patients were eligible for study inclusion if they (1) were at least 18 years old, (2) had been diagnosed with non-valvular AF, and (3) received continuous oral anti-coagulants (either VKAs or DOACs) for at least 3 months before the date of study inclusion. A sample size of 100 patients per country was deemed sufficient to estimate parameter coefficients, given a rule of thumb for discrete choice experiments [17]. The study proposal and survey design were deemed exempt from the Medical Research Involving Human Subjects Act by the institutional review board of the University of Twente. The board judged whether the study met the criteria for medical ethical review in the Netherlands. The market agency made sure that the way in which respondent background characteristics were collected in the survey met the data privacy regulations in each country.

\subsection{Statistical Analysis}

\subsubsection{Background Characteristics}

Descriptive statistics were used to study patients' sociodemographic and treatment-related characteristics. Educational level was categorized as low, medium, or high using the International Standard Classification of Education (ISCED [18]. Annual stroke risk was calculated using the $\mathrm{CHA}_{2} \mathrm{DS}_{2}$-VASc risk score, and annual bleeding risk was calculated using the HAS-BLED score, based on selfreported co-morbidities [19]. Potential statistical differences between VKA and DOAC users were tested using Pearson's Chi squared test and the independent $t$ tests in SPSS. $P$ values $<0.05$ were considered statistically significant.

\subsubsection{Choice Data}

The discrete choice data were analyzed using a mixed logit model and a latent class model. In the discrete choice experiment, six respondents were straight-liners, which means that they either chose the left or right scenario in all questions. Data analysis with and without these respondents yielded similar results for both models, and the data from all respondents was included in the analysis.

2.5.2.1 Mixed Logit Model (MLA) The aim of the MLA was to quantify the average impact of attribute levels (coefficients $\beta$ ) on the preference for treatment and to establish the individual specific variation in preferences between respondents [20]. The mixed logit model is described in the article by Hauber et al. [13]. MLA was performed using R Statistical Software [21].

For each attribute level, both the mean coefficient $(\beta)$ and the standard deviation (SD) of the effects across the sample were estimated. Dummy coding was used for the attributes in the analysis, where the reference level has a value of zero, 


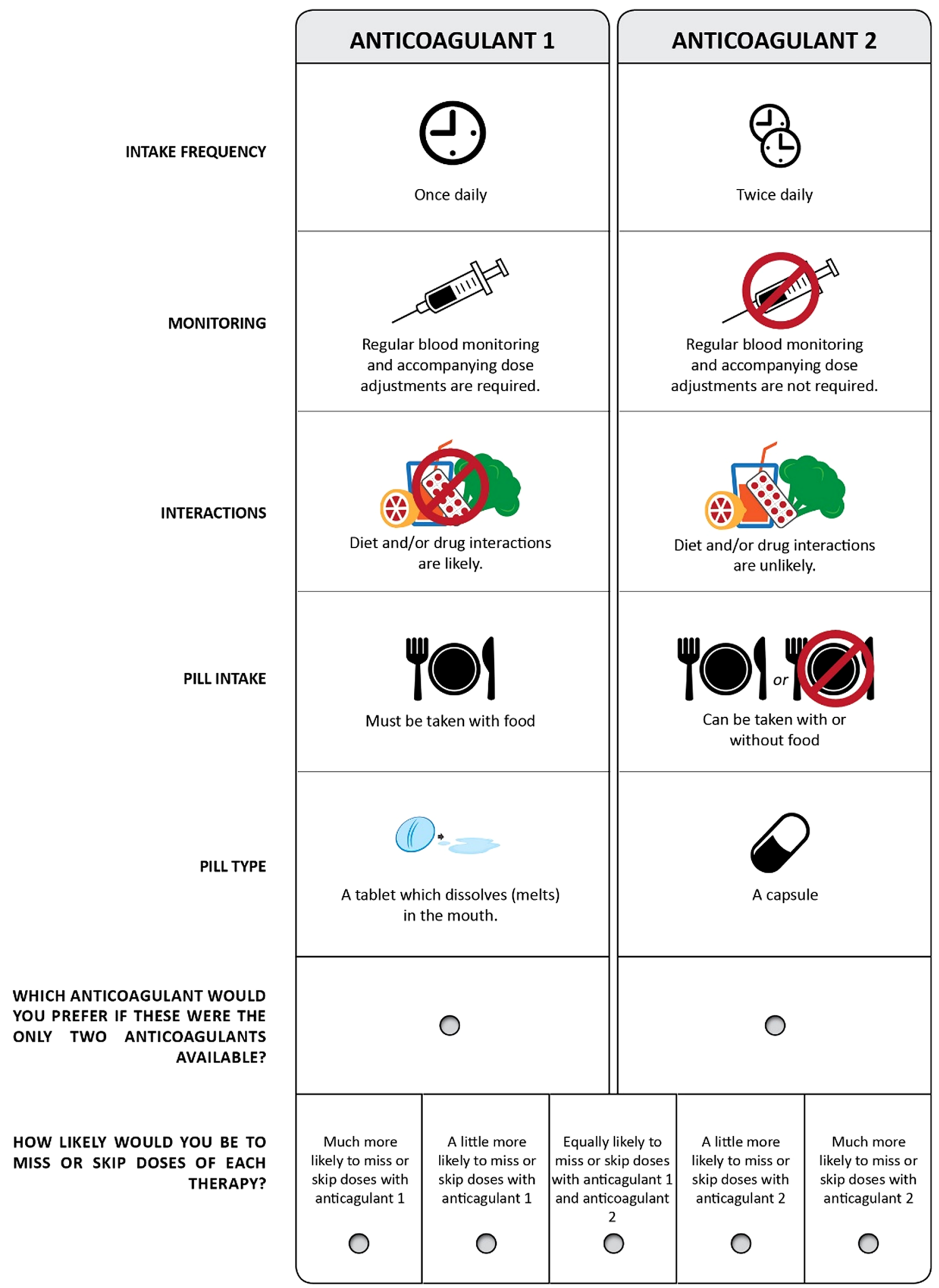

Fig. 1 Example of a discrete choice question. The five attributes and their levels were organized into pairs of hypothetical medication profiles. Both options include all attributes: intake frequency, coagulation monitoring, drug/food interaction, pill intake, and pill type, but differ with regard to the attribute levels. Patients were asked repeat- edly which anti-coagulant they would choose if the two profiles in each question were the only two anti-coagulants available and equal effectiveness and safety of the drugs presented in the profile was assumed 
except for the effect of country in the multivariate analysis. The statistical tests associated with the regression coefficient reflect the statistical significance of the difference between each level and the reference level [13]. Attribute importance (AI), i.e., the impact on the value of a treatment option of changing one attribute level to another level of the same attribute regarding the overall preference for treatment, was calculated using profile-based normalization as described by Gonzalez et al. [22]. In profile-based normalization, AI is presented as the proportion of the change in utility in going from the absolute worst to the absolute best treatment profile. Mixed logit models were estimated with the assumption of normalized coefficient distribution. The fit of the model was judged based on the log likelihood (LL). The fit of the model without correlation (LL 3444.4) was better than the model with correlation (LL 3432.5) between coefficients. Univariate and multivariate models were estimated. The multivariate model with the co-variates gender, current DOAC, adherence, and country of residence had the highest fit (LL 3366.4).

2.5.2.2 Latent Class Model (LCA) The main aim of the LCA was to further examine preference heterogeneity in the respondent data and to link differences in preferences to differences in background characteristics. Preference heterogeneity is the degree to which preferences for anti-coagulation therapy vary between respondents, i.e., the extent to which different respondents have different preferences. Scale heterogeneity reflects the variation in the respondents' choice consistency [23]. Highly consistent respondents have a lower error variance than less consistent respondents [24]. It is assumed that highly consistent respondents have stronger preferences or, in other words, are more certain of their preferences. The Scale-Adjusted Latent Class (SALC) model enables a simultaneous estimation of preference and scale heterogeneity [25]. Using the SALC approach, respondents are described as having a probability of being in a latent preference class, which distinguishes their preferences for therapy from other respondents in another class; simultaneously, they are described as having a probability of being in a latent scale class, which distinguishes the consistency in (the "strength of") their preferences. The number of scale (sClass) and preference (pClass) classes in this study were determined using an iterative approach, where the ultimate model was selected based on model fit [the Bayesian information criterion (BIC)] and interpretability of the model (fewer classes are preferred). Age, gender, country, current coagulation therapy, and drug adherence were added as covariates to study whether these observed factors influenced preferences.

\section{Results}

\subsection{Background Characteristics}

A total of 508 patients ( $62 \%$ male) from five European countries completed the survey. Patients were aged $57.2 \pm 15.0$ years. With regard to type of pill used, $37 \%$ $(n=188)$ of patients took DOACs and 63\% $(n=320)$ VKAs. DOAC users had a lower educational level and a lower annual household income, were less often employed and more often male, and they had a higher daily pill burden ( 6.5 pills vs. 5.4 pills) as compared to VKA users. Of the current DOAC users, almost $40 \%$ had used a VKA in the past. VKA and DOAC users were similar in their risk profiles for stroke and bleeding. The sociodemographic and treatment-related characteristics of the patients are presented in Table 1.

\subsection{Self-Reported Adherence}

Self-reported adherence in the study sample was high. A total of $57.7 \%(n=293)$ of respondents indicated that they rarely missed or skipped a dose (high adherence), $34.3 \%$ $(n=174)$ occasionally missed or skipped a dose (high to moderate adherence), and $7.5 \%(n=38)$ sometimes missed or skipped a dose (moderate adherence). Only three respondents $(0.6 \%)$ reported that they often forget or skip a dose. No difference was found in self-reported adherence between DOAC users and VKA users $\left(\mathrm{Chi}^{2}=4.1 ; p=0.249\right)$.

\subsection{Results from the MLA}

All attributes had a significant impact on the choice of therapy in the mixed logit model $(p<0.05)$. In Table 2 , it can be seen that intake without food $(B$ 0.197) is preferred to intake with food, once-daily intake $(B 0.517)$ is preferred to twice-daily intake, no need for International Normalized Ratio (INR) monitoring ( $B$ 0.806) is preferred to a need for INR monitoring, a tablet $(B$ 0.121) is preferred to a capsule, and unlikely drug/food interactions $(B 0.456)$ are preferred to likely drug/food interactions $(p<0.05)$. The difference in preference between a melting tablet and a capsule was not significant $(p=0.123)$. The need for INR monitoring had the greatest impact on preference for therapy $(\mathrm{AI}=37 \%$; Table 2). The second most important attribute was intake frequency (AI 27\%). The results of the MLA showed significant variation in preferences, as represented by the high SD for the need for coagulation monitoring, intake frequency, and drug/food interactions (SD significant $p<0.000$ ). In the multivariate MLA model, women had stronger preference for tablets compared to capsules than men, and men have stronger preferences for no monitoring than women (Appendix 1 in the electronic supplementary material). With 
Table 1 Characteristics of the study sample. Data reported as $n(\%)$, unless otherwise marked

\begin{tabular}{|c|c|c|c|c|}
\hline & All patients $(n=508)$ & DOAC users $(n=188)$ & VKA users $(n=320)$ & $P$ value \\
\hline Gender, male & $315(62.0)$ & $127(67.6)$ & $188(58.8)$ & $P=0.05$ \\
\hline Mean age, years \pm SD & $57.2 \pm 15.0$ & $57.0 \pm 15.4$ & $57.4 \pm 14.7$ & $P=0.80$ \\
\hline Country & & & & $P=0.001$ \\
\hline United Kingdom & $100(19.7)$ & $38(38.0)$ & $62(62.0)$ & \\
\hline Germany & $100(19.7)$ & $43(43.0)$ & $57(57.0)$ & \\
\hline France & $103(20.3)$ & $51(49.5)$ & $52(50.5)$ & \\
\hline Italy & $103(20.3)$ & $30(29.1)$ & $73(70.9)$ & \\
\hline Spain & $102(20.1)$ & $26(25.5)$ & $76(74.5)$ & \\
\hline Educational level & & & & $P=0.05$ \\
\hline Low & $77(15.2)$ & $34(18.1)$ & $43(13.4)$ & \\
\hline Medium & $301(59.3)$ & $119(63.3)$ & $182(56.9)$ & \\
\hline High & $130(25.6)$ & 35 (18.6) & $95(29.7)$ & \\
\hline Employment status & & & & $P<0.001$ \\
\hline Employed (full/part-time) & $223(43.9)$ & $56(29.8)$ & $167(52.2)$ & \\
\hline Unemployed & $24(4.7)$ & $8(4.3)$ & $16(5.0)$ & \\
\hline Retired & $261(51.4)$ & $124(66.0)$ & $137(42.8)$ & \\
\hline Living situation & & & & $P=0.22$ \\
\hline Independent & $494(97.2)$ & $187(99.5)$ & 307 (95.9) & \\
\hline Care home/nursing home & $4(0.8)$ & $0(0.0)$ & $4(1.3)$ & \\
\hline Other & $10(2.0)$ & $1(0.5)$ & $9(2.8)$ & \\
\hline Annual household income, $€$ & & & & $P=0.01$ \\
\hline$€ 0-€ 15,000$ & $81(15.9)$ & $34(18.1)$ & $47(14.7)$ & \\
\hline$€ 15,001-€ 25,000$ & $112(22.0)$ & $45(23.9)$ & $67(20.9)$ & \\
\hline$€ 25,001-€ 50,000$ & $164(32.3)$ & $62(33.0)$ & $102(31.9)$ & \\
\hline$€ 50,001-€ 75,000$ & $54(10.6)$ & $23(12.2)$ & $31(9.7)$ & \\
\hline$€ 75,000+$ & $66(13.0)$ & $9(4.8)$ & $57(17.8)$ & \\
\hline I do not wish to answer & $31(6.1)$ & $15(8.0)$ & $16(5.0)$ & \\
\hline \multicolumn{5}{|l|}{ Current OAC use } \\
\hline Total no. of patients on VKA & $320(63.0)$ & & & \\
\hline Warfarin & $127(25.0)$ & - & $127(39.7)$ & \\
\hline Acenocoumarol & $52(10.2)$ & - & $52(16.3)$ & \\
\hline Phenprocoumon & $76(15.0)$ & - & $76(23.8)$ & \\
\hline Fluindione & $65(12.8)$ & - & $65(20.3)$ & \\
\hline Total no. of patients on DOAC & $188(37.0)$ & & & \\
\hline Dabigatran etexilate & $39(7.7)$ & $39(20.7)$ & - & \\
\hline Rivaroxaban & $87(17.1)$ & $87(46.3)$ & - & \\
\hline Apixaban & $51(10.0)$ & $51(27.1)$ & - & \\
\hline Edoxaban & $11(2.2)$ & $11(5.9)$ & - & \\
\hline Time since AF diagnosis, years & & & & $P=0.001$ \\
\hline Mean \pm SD & $3.2 \pm 1.2$ & $3.1 \pm 1.3$ & $3.3 \pm 1.1$ & \\
\hline$<1$ year & $37(7.3)$ & $20(10.6)$ & $17(5.3)$ & \\
\hline $1-5$ years & $284(55.9)$ & $99(52.7)$ & $185(57.8)$ & \\
\hline$>5$ years & $184(36.2)$ & $66(35.1)$ & $118(36.9)$ & \\
\hline Don’t know & $3(0.6)$ & $3(1.6)$ & $0(0.0)$ & \\
\hline Total time on current OAC, months/years & & & & $P<0.001$ \\
\hline Mean \pm SD & $3.0 \pm 0.8$ & $3.0 \pm 0.8$ & $3.1 \pm 0.8$ & \\
\hline$<1$ year & $123(24.2)$ & $56(29.8)$ & $68(21.2)$ & \\
\hline 1 year-5 years & $251(49.4)$ & $104(55.3)$ & $145(45.3)$ & \\
\hline$>5$ years & $126(24.8)$ & $25(13.3)$ & $102(31.9)$ & \\
\hline I do not remember & $8(1.6)$ & $3(1.6)$ & $5(1.6)$ & \\
\hline
\end{tabular}


Table 1 (continued)

All patients $(n=508) \quad$ DOAC users $(n=188) \quad$ VKA users $(n=320) \quad P$ value

Have you switched from VKA to DOAC?

\begin{tabular}{|c|c|c|c|c|}
\hline No & $114(22.4)$ & $114(60.6)$ & - & \\
\hline Yes & $74(14.6)$ & $74(39.4)$ & - & \\
\hline HAS-BLED score & & & & $P=0.88$ \\
\hline Mean \pm SD & $2.4 \pm 1.5$ & $2.4 \pm 1.5$ & $2.4 \pm 1.5$ & \\
\hline HAS-BLED score 0-1 (“low” risk) & $150(29.5)$ & $57(30.3)$ & $93(29.1)$ & \\
\hline HAS-BLED score 2 (“moderate" risk) & $128(25.2)$ & $47(25.0)$ & $81(25.3)$ & \\
\hline HAS-BLED score $\geq 3$ (“high” risk) & $230(45.3)$ & $84(44.7)$ & $146(45.6)$ & \\
\hline $\mathrm{CHA}_{2} \mathrm{DS}_{2}$-VASc score & & & & $P=0.57$ \\
\hline Mean \pm SD & $2.4 \pm 1.4$ & $2.4 \pm 1.4$ & $2.4 \pm 1.5$ & \\
\hline $\mathrm{CHA}_{2} \mathrm{DS}_{2}$-VASc score 0,1 (“low” risk) & $154(30.3)$ & $57(30.3)$ & $97(30.3)$ & \\
\hline $\mathrm{CHA}_{2} \mathrm{DS}_{2}$-VASc score 2 ("moderate" risk) & $130(25.6)$ & $51(27.1)$ & $79(24.7)$ & \\
\hline $\mathrm{CHA}_{2} \mathrm{DS}_{2}$-VASc score $\geq 3$ (“high" risk) & $224(44.1)$ & $80(42.6)$ & $144(45.0)$ & \\
\hline Total number of pills taken each day ("pill burden") & & & & $P=0.01$ \\
\hline Mean \pm SD (range) & $5.8 \pm 5.1(0.5-50)$ & $6.5 \pm 4.4(1-20)$ & $5.4 \pm 5.4(0.5-50)$ & \\
\hline
\end{tabular}

The $\mathrm{CHA}_{2} \mathrm{DS}_{2}$-VASc [Cardiac failure or dysfunction, Hypertension, Age $\geq 75$ years (Doubled), Diabetes, Stroke (Doubled)-Vascular disease, Age 65-74 years and Sex category (Women)] risk index estimates stroke risk in AF patients. Low-risk patients may not require anti-coagulation; moderate-risk patients should consider anti-coagulation, and high-risk patients are an anti-coagulation candidate. The HAS-BLED (Hypertension, Abnormal renal/liver function, Stroke, Bleeding history or predisposition, Labile INR, Elderly, and Diabetes) risk index estimates bleed risk in $\mathrm{AF}$ patients. Low-risk patients should consider anti-coagulation; in moderate-risk patients, anti-coagulation can be considered, but for high-risk patients, alternatives to anti-coagulation should be considered

$A F$ atrial fibrillation, $D O A C$ direct-acting oral anti-coagulant, $O A C$ oral anti-coagulant, $S D$ standard deviation, $V K A$ vitamin $\mathrm{K}$ antagonist

Table 2 Patient preference for attributes of convenience (mixed logit analysis without correlations)

\begin{tabular}{llllllll}
\hline Attribute & Level & $B$ & SE & Sig (SE) & SD & Sig (SD) & Importance (\%) \\
\hline Pill intake & Without food & 0.197 & 0.037 & 0.000 & 0.275 & 0.001 & 9 \\
& With food* & 0.000 & & & & & \\
Intake frequency & Once daily & 0.571 & 0.052 & 0.000 & 0.836 & 0.000 & 27 \\
& Twice daily* & 0.000 & & & & & \\
Pill type & Tablet & 0.121 & 0.050 & 0.016 & 0.305 & 0.001 & 6 \\
& Melting tablet & 0.079 & 0.051 & 0.123 & 0.290 & 0.005 & \\
Coagulation monitoring & Capsule* & 0.000 & & & & & \\
& Not required & 0.806 & 0.076 & 0.000 & 1.542 & 0.000 & 37 \\
& Required* & 0.000 & & & & & \\
Drug/food interactions & Unlikely & 0.456 & 0.053 & 0.000 & 0.886 & 0.000 & 21 \\
& Likely* & 0.000 & & & & &
\end{tabular}

Part-worth utility coefficients $(B)$ and variation within part-worth utility coefficients (SD) of attribute levels of convenience of AF therapy

$A F$ atrial fibrillation, $S D$ standard deviation, $S E$ standard error

*Reference level. Higher part-worth utility indicates higher preference for this level regard to the effect of current anti-coagulant therapy, VKA users have stronger preferences for once-daily pills and tablets, while DOAC users have a stronger preference for no monitoring.

With regard to the impact of country, we see that for every attribute, there is at least one country in which patients have significantly different preferences. For instance, although patients from Germany preferred no monitoring of coagulation, this preference was less strong than that of respondents from Italy. Patients from the UK had a stronger preference for once-daily pill intake than respondents from other countries. Likewise, patients from the UK have a stronger preference for drugs in which drug/food interactions are unlikely, while in patients from Spain, this preference is less strong than the average. 


\subsection{Results from the LCA}

The analysis was performed for between two and six preference classes and zero and six scale classes. Adding two scale classes to the model improved model fit; the difference with a three-scale class model was negligible (Appendix 3 in the electronic supplementary material). The five-preference class model had the best fit when the scale factor was not taken into account, but the 2-sClass-4-pClass model had a better fit than the 2-sClass-5-pClass model. Thus, the 2-sClass-4-pClass model was considered the optimal model based on model fit and interpretability $(\mathrm{BIC}=68.6$; $\mathrm{L} 2=55.8)$. The two scale classes were significantly different $(p<0.000)$. Of the 508 patients in our sample, $44 \%$ were members of the strong preference class and $56 \%$ the weak preference class (Table 3). The scale factor was 14 , which means that the part-worth coefficients in the model of the strong preference class were 14 times as large as those of the weak preference class.

There was significant preference heterogeneity for intake frequency, the need for coagulation monitoring, and drug/ food interactions $(p<0.05)$ across classes. Current anticoagulant therapy, country of residence, and self-reported adherence were correlated with class membership $(p<0.05)$. Full details of the 2-sClass-4-pClass LCA are provided in Appendix 2 (see the electronic supplementary material).

Scale class membership was about equally divided over respondents in all countries, although a relative higher percentage of the patients from Spain fell in the weak preference class. Membership of the weak preference class was also somewhat more likely for VKA users, while membership to the strong preference class was somewhat more likely for DOAC users.

For two of the attributes, the level order was as expected in all classes. Respondents prefer to take pills once daily and without food. All classes except class 4 prefer no coagulation monitoring. For the attribute drug/food interaction, class 1 and 3 prefer drugs for which drug/food interactions are likely. For the attribute pill type, no prior expectations on ordering existed. The results indicate that this attribute does not influence preferences.

Patients in the "no coagulation monitoring group" (class $1 ; 57 \%)$ attached the highest relative importance to the need for monitoring (B 2.802; AI 68\%), but also considered interactions between drugs important (AI 15\%) (Table 3). Class members were more likely to be DOAC users (19\% more than expected based on class size) and less likely to be VKA users (11\% less than expected) (Appendix 2).

Of the respondents, $19 \%$ were members of the "balanced" preference class (class 2). They attached the most importance to once-daily intake frequency (AI 31\%), equal importance to intake without food and pill type (AI 23\%), and lesser importance to coagulation monitoring (AI 13\%) and drug/food interactions (AI 11\%). VKA users were more likely to be members of this class.

Table 3 Patient preference patterns for attributes of convenience (latent class analysis)

\begin{tabular}{|c|c|c|c|c|c|c|}
\hline \multicolumn{2}{|l|}{ Class size } & \multirow{2}{*}{$\begin{array}{l}\text { Class } 1(\%) \\
25\end{array}$} & \multirow{2}{*}{$\begin{array}{l}\text { Class } 2(\%) \\
9\end{array}$} & \multirow{2}{*}{$\begin{array}{l}\text { Class } 3(\%) \\
7\end{array}$} & \multicolumn{2}{|l|}{ Class $4(\%)$} \\
\hline & sClass 1 (strong) & & & & 3 & 44 \\
\hline & sClass 2 (weak) & 32 & 11 & 9 & 4 & 56 \\
\hline & Total & 57 & 20 & 16 & 7 & 100 \\
\hline Attribute & Level & Class 1 & Class 2 & Class 3 & Class 4 & $P$ value \\
\hline \multirow[t]{2}{*}{ Pill intake } & Without food & 0.208 & 7.988 & 0.129 & 0.337 & 0.057 \\
\hline & With food* & 0.000 & 0.000 & 0.000 & 0.000 & \\
\hline \multirow[t]{2}{*}{ Intake frequency } & Once daily & 0.289 & 10.966 & 16.220 & 0.267 & 0.002 \\
\hline & Twice daily* & 0.000 & 0.000 & 0.000 & 0.000 & \\
\hline \multirow[t]{3}{*}{ Pill type } & Tablet & 0.127 & -0.205 & 0.344 & 0.905 & 0.400 \\
\hline & Melting tablet & -0.080 & -7.987 & 0.431 & 0.291 & \\
\hline & Capsule* & 0.000 & 0.000 & 0.000 & 0.000 & \\
\hline \multirow[t]{2}{*}{ Coagulation monitoring } & Not required & 2.802 & 4.504 & 0.667 & -3.207 & 0.000 \\
\hline & Required* & 0.000 & 0.000 & 0.000 & 0.000 & \\
\hline \multirow[t]{3}{*}{ Drug/food interactions } & Unlikely & -0.616 & 3.793 & -18.721 & -0.173 & 0.000 \\
\hline & Likely* & 0.000 & 0.000 & 0.000 & 0.000 & \\
\hline & Overall & Wald & $P$ value & & & \\
\hline sClass 1 & 0.0000 & 79.778 & 0.000 & & & \\
\hline sClass2 & -2.6586 & & & & & \\
\hline
\end{tabular}

sClass number of scale class 
Sixteen percent of respondents (class 3) fell into the "once daily, interactions likely" pattern. This means that they attach about equal importance to once-daily pill intake (AI 45\%) and prefer pills for which drug/food interactions are likely (AI 52\%).

Finally, 7\% of respondents (class 4) were members of the "coagulation monitoring preferred pattern." These patients also attached highest importance to the need for monitoring (67\%); however, in contrast to the patients in class 1, they preferred to be monitored. Only $3 \%$ of the DOAC users and $10 \%$ of the VKA users were in this class. Thus, membership to this class was more likely for VKA users.

The least adherent patients $(n=38)$ were somewhat overrepresented in class 1 , while moderately adherent patients $(n=174)$ were overrepresented in class 2.

There was also a significant impact of country of residence $(p<0.004)$ on the likelihood of being in a preference class. Most noticeably, respondents from the UK and Germany were less likely to be in the "no coagulation monitoring" group, while respondents from Italy were most likely to be in this class. Respondents from Germany were more likely to be in the "once daily, interactions likely" class (class 3 ) than expected based on class size.

No significant impact of pill burden, gender, and age on preference class membership was found.

\section{Discussion}

This study in 508 patients with non-valvular AF from five European countries explored the heterogeneity in preferences for convenience of anti-coagulation therapy and assessed whether preference heterogeneity can be explained by background characteristics. The results of this study demonstrate significant preference heterogeneity among respondents to the survey, both in the MLA and in the LCA. While many preference studies focusing on convenience of anticoagulant therapy exist, the results of our study increase the insight into patient heterogeneity in preferences compared to current literature. For instance, while Andrade et al. found that the need for coagulation monitoring is more important to patients than the intake frequency of the drug [26], the study of Böttger et al. found the opposite to be true [27]. The results of our study indicate that four relevant preference patterns exist. Six out of ten patients focused on the need for monitoring as the most decisive attribute of treatment and preferred no regular coagulation monitoring; these patients were more likely to be current DOAC users and the least adherent to therapy. One out of five patients considered most attributes in their choice; these patients were more likely current VKA users and moderately adherent. The third group, representing one out of six patients, preferred once-daily pill intake but—surprisingly—also preferred scenarios with a high likelihood of drug/food interaction; these patients were more likely to have a low adherence. While in the three aforementioned classes patients preferred not to be monitored, a small group of patients (one out of 14 patients) preferred to undergo regular coagulation monitoring; these patients were more likely to currently use VKA and reported high adherence.

In this study, MLA and LCA were used to study preference heterogeneity. Heterogeneity in the study sample could be identified from the large SDs of the utility coefficients in the MLA. The results of both analyses align in the sense that the attributes with large variation in the MLA were also the attributes that distinguished the patients in the LCA. The covariates that significantly impacted preferences were similar in both models, although more significant relationships were determined in the MLA. However, MLA with correlations either results in a large number of different preference models (one for each covariate) or in a very large model that can be difficult to interpret. The LCA allowed us to include multiple covariates and to study their impact simultaneously, to distinguish a limited number of very specific preference patterns, and to present these in a more intuitive and comprehensible way.

\subsection{Potential Clinical Implications}

The results of this study have potential clinical implications. First, the study results show that current therapy is a covariate that is associated with class membership. The majority of patients are more likely to prefer treatment characteristics that align with their current therapy. We know that $40 \%$ of current DOAC users switched from VKA to DOAC in the past; however, we did not ask current VKA users whether they switched from DOAC to VKA. Therefore, we could not include switching as a covariate, and the results of the study do not offer insight into whether patients prefer the characteristics of the drug they have or have switched to the drug of their preference in the past. Some incongruence between the preferred and the actual anti-coagulation therapy also exists. For instance, membership to class 1 and 2, in which patients preferred no regular coagulation monitoring, included a significant percentage of current VKA users. On the other hand, few patients in the group that prefers monitoring are currently on DOACs.

A second interesting finding of our study is the observed relationship between class membership and self-reported adherence to therapy. While no causal relationship can be drawn between class membership and self-reported adherence, it is intriguing to see that patients who prefer regular coagulation monitoring are more likely to report high adherence, while those who do not prefer monitoring are more likely to report a low adherence. As previously reported, the feedback mechanism that results from regular monitoring emphasizes adherence to therapy [28]. 
Third, the impact of country of residence on the preferences for treatment is both interesting and challenging to explain. Previous studies also identified differences in preference between European countries. For instance, patients in Germany were more likely to prefer no monitoring than patients in Italy. Preference for monitoring might be related to the ability for the patient to access the health care system in the different countries. Monitoring might be viewed as an opportunity to have regular contact with the physician, in countries were access is more difficult.

Finally, the results of our study suggest that many respondents focused mostly on one attribute in stating their preference for an anti-coagulation drug. Either intake frequency or the need for monitoring are dominant. In the survey, respondents consistently chose the scenario with either once-daily pill intake, no need for monitoring or the need for monitoring. Our data suggest that only for a smaller group of patients (class 2) did the choice of therapy involve multiple attributes of therapy. These patients either trade between levels of the different scenarios in the choice set or use a lexicographic decision rule, where the scenario with the most desirable characteristics is chosen.

\subsection{Limitations}

As with any stated preference survey, the results of this study are highly dependent on the choice of attributes and the operationalization of attributes. We have tried to match the most commonly used attributes and level descriptions in literature available at the time of our study [16, 26, 27], but the way in which we operationalized some of the attributes has likely influenced the results. Most noticeably, class 1 and 3 patients seem to have misunderstood the picture we used to present likelihood of drug/food interactions. This misunderstanding did not occur during pilot testing, but it is likely that the attention of respondents during an online survey is lower than during pilot testing, when the researcher was present. Another limitation of our study is that while we performed external validity tests, by doing pilot testing and involving experts, we did not perform any internal validity test besides looking for straight liners in our data set [29].

This study gives insight into the preferences of patients for certain treatment outcomes. However, it does not provide information on why these preferences exist. For example, monitoring, which is needed with a VKA, allows for some control of the blood anti-coagulation level. Based on the anti-coagulant level, VKA dosing might be adjusted. Some patients might associate monitoring with an increased feeling of control and feedback on drug adherence, or even increased drug effectiveness and thus lower risk of stroke. Others might find the monthly or bi-weekly appointments burdensome because of the disruption to work or personal life and therefore prefer not to be monitored. However, it is also possible that patients might assume that because a drug has the need for monitoring, it is less effective or less safe than a drug that does not require monitoring. Based on this study, we can only conclude that most patients prefer drugs that do not require monitoring, but not the reasons for this preference. The same is true for the other attributes.

Our study population was younger than the average population of all AF patients in clinical practice. This may have resulted from the fact that we recruited participants on the internet, using online panels. However, within the age groups included in the study, age did not influence class membership, which indicates that like younger patients, the majority of elderly patients prefer once-daily treatment without a need for coagulation monitoring. We assessed patients from the five European countries that have the largest population sizes, which resulted in an underrepresentation of Eastern and Northern Europe countries. We intentionally did not include attributes related to probability of stroke and bleeding in our attribute set because we were interested in the tradeoff between convenience attributes and feared they would be dominated by effectiveness and safety concerns, as was shown earlier [8,9], but it would be very interesting to study whether there are different classes of patients with regard to the trade-off between benefit, risk, and convenience.

\section{Conclusions}

This study demonstrated significant preference heterogeneity among patients with AF and linked different classes of preference to specific background characteristics. Country of residence and currently prescribed therapy influenced patient preferences in both the MLA and LCA models. Further studies should focus on the relationship between preference and therapy adherence and on ways to turn knowledge about individual preference into support tools for preferencesensitive treatment decisions.

Acknowledgements We would like to thank Melissa Vaanholt for her effort during the design and analysis of the results phases of this study, and the clinicians that provided feedback on the clinical relevance of the attributes and the validity of the survey design.

Author Contributions JvT was responsible for project management and has contributed to the design of the survey, statistical data analysis, and interpretation of the data. $\mathrm{CO}-\mathrm{G}$ was responsible for statistical analysis and interpretation of the data. MW contributed to survey design and was responsible for pilot testing of the survey. $\mathrm{CvB}$ has contributed to clinical interpretation of the data. All authors have contributed to the writing of the manuscript.

\section{Compliance with Ethical Standards}

Funding This study was funded by Daiichi Sankyo Europe GmbH (Munich, Germany). 
Conflict of interest J. van Til, C. Oudshoorn-Groothuis, M. Weernink, and $\mathrm{C}$. von Birgelen declare no conflict of interest.

Data Availability Statement The data set from this study is available from the authors upon request.

Open Access This article is licensed under a Creative Commons Attribution-NonCommercial 4.0 International License, which permits any non-commercial use, sharing, adaptation, distribution and reproduction in any medium or format, as long as you give appropriate credit to the original author(s) and the source, provide a link to the Creative Commons licence, and indicate if changes were made. The images or other third party material in this article are included in the article's Creative Commons licence, unless indicated otherwise in a credit line to the material. If material is not included in the article's Creative Commons licence and your intended use is not permitted by statutory regulation or exceeds the permitted use, you will need to obtain permission directly from the copyright holder.To view a copy of this licence, visit http://creativecommons.org/licenses/by-nc/4.0/.

\section{References}

1. Wilke T, Groth A, Mueller S, Pfannkuche M, Verheyen F, Linder $\mathrm{R}$, et al. Incidence and prevalence of atrial fibrillation: an analysis based on 8.3 million patients. Europace. 2013;15(4):486-93.

2. Pistoia F, Sacco S, Tiseo C, Degan D, Ornello R, Carolei A. The epidemiology of atrial fibrillation and stroke. Cardiol Clin. 2016;34(2):255-68.

3. Hirschl M, Kundi M. Safety and efficacy of direct acting oral anticoagulants and vitamin $\mathrm{K}$ antagonists in nonvalvular atrial fibrillation - a network meta-analysis of real-world data. VASA Zeitschrift fur Gefasskrankheiten. 2019;48(2):134-47.

4. Almutairi AR, Zhou L, Gellad WF, Lee JK, Slack MK, Martin $\mathrm{JR}$, et al. Effectiveness and safety of non-vitamin K antagonist oral anticoagulants for atrial fibrillation and venous thromboembolism: a systematic review and meta-analyses. Clin Ther. 2017;39(7):1456-78.e36.

5. Ahmad Y, Lip GYH. Stroke prevention in atrial fibrillation: concepts and controversies. Curr Cardiol Rev. 2012;8(4):290-301.

6. Burn J, Pirmohamed M. Direct oral anticoagulants versus warfarin: is new always better than the old? Open Heart. 2018;5(1):e000712.

7. Heidbuchel H, Verhamme P, Alings M, Antz M, Diener HC, Hacke W, et al. Updated European Heart Rhythm Association Practical Guide on the use of non-vitamin K antagonist anticoagulants in patients with non-valvular atrial fibrillation. Europace. 2015;17(10):1467-507.

8. Wilke T, Bauer S, Mueller S, Kohlmann T, Bauersachs R. Patient preferences for oral anticoagulation therapy in atrial fibrillation: a systematic literature review. Patient. 2017;10(1):17-37.

9. Loewen PS, Ji AT, Kapanen A, McClean A. Patient values and preferences for antithrombotic therapy in atrial fibrillation. A narrative systematic review. Thromb Haemostas. 2017;117(6):1007-22.

10. Weernink MGM, Vaanholt MCW, Groothuis-Oudshoorn CGM, von Birgelen C, MJ IJ, van Til JA. Patients' priorities for oral anticoagulation therapy in non-valvular atrial fibrillation: a multi-criteria decision analysis. Am J Cardiovasc Drugs. 2018;18(6):493-502.

11. Groothuis-Oudshoorn CGM, Flynn TN, Yoo HI, Magidson J, Oppe M. Key issues and potential solutions for understanding healthcare preference heterogeneity free from patient-level scale confounds. Patient Patient Center Outcomes Res. 2018;11(5):463-6.

12. Bridges JF, Hauber AB, Marshall D, Lloyd A, Prosser LA, Regier DA, et al. Conjoint analysis applications in health-a checklist: a report of the ISPOR Good Research Practices for Conjoint Analysis Task Force. Value Health. 2011;14(4):403-13.

13. Hauber AB, González JM, Groothuis-Oudshoorn CGM, Prior T, Marshall DA, Cunningham C, et al. Statistical methods for the analysis of discrete choice experiments: a report of the ISPOR Conjoint Analysis Good Research Practices Task Force. Value Health. 2016;19(4):300-15.

14. Vaanholt MCW, Weernink MGM, von Birgelen C, GroothuisOudshoorn CGM, MJ IJ, van Til JA. Perceived advantages and disadvantages of oral anticoagulants, and the trade-offs patients make in choosing anticoagulant therapy and adhering to their drug regimen. Patient Educ Counsel. 2018;101(11):1982-9.

15. Douketis JD, Spyropoulos AC, Kaatz S, Becker RC, Caprini JA, Dunn AS, et al. Perioperative bridging anticoagulation in patients with atrial fibrillation. N Engl J Med. 2015;373(9):823-33.

16. Ghijben PL, Zavarsek S. Preferences for oral anticoagulants in atrial fibrillation: a best-best discrete choice experiment. PharmacoEconomics. 2014;32(11):1115-27.

17. Johnson FR, Lancsar E, Marshall D, Kilambi V, Mühlbacher A, Regier DA, et al. Constructing experimental designs for discretechoice experiments: report of the ISPOR conjoint analysis experimental design good research practices task force. Value Health. 2013;16(1):3-13.

18. Statistics UIf. International Standard Classification of Education: ISCED 2011. Montreal: UIS; 2012. p. 85.

19. Borre ED, Goode A, Raitz G, Shah B, Lowenstern A, Chatterjee $\mathrm{R}$, et al. Predicting thromboembolic and bleeding event risk in patients with non-valvular atrial fibrillation: a systematic review. Thromb Haemost. 2018;118(12):2171-87.

20. McFadden D, Train K, McFadden D. Train KMixed MNL models for discrete response. J Appl Econometr. 2000;15:447-70.

21. Croissant Y. Package 'mlogit' 2019 [R package version 0.2-4]. https://cran.r-project.org/web/packages/mlogit/mlogit.pdf.

22. Gonzalez JM. A guide to measuring and interpreting attribute importance. Patient. 2019;12(3):287-95.

23. Swait J, Louviere J. The role of the scale parameter in the estimation and comparison of multinomial logit models. J Mark Res. 1993;30(3):305-14.

24. Vass CM, Wright S, Burton M, Payne K. Scale heterogeneity in healthcare discrete choice experiments: a primer. Patient PatientCenter Outcomes Res. 2018;11(2):167-73.

25. Vermunt JK, Magidson J. Latent class analysis with sampling weights: a maximum-likelihood approach. Sociol Methods Res. 2007;36(1):87-111.

26. Andrade JG, Krahn AD, Skanes AC, Purdham D, Ciaccia A, Connors $\mathrm{S}$. Values and preferences of physicians and patients with nonvalvular atrial fibrillation who receive oral anticoagulation therapy for stroke prevention. Can J Cardiol. 2016;32(6):747-53.

27. Böttger BT-WIM, Bauersachs R, Kohlmann T, Wilke T. Preferences for anticoagulation therapy in atrial fibrillation: the patients' view. J Thromb Thrombol. 2015;40(4):406-15.

28. Barcellona D, Luzza M, Battino N, Fenu L, Marongiu F. The criteria of the Italian Federation of Thrombosis Centres on DOACs: a "real world" application in nonvalvular atrial fibrillation patients already on vitamin $\mathrm{K}$ antagonist. Intern Emerg Med. 2015;10(2):157-63.

29. Johnson FR, Yang J-C, Reed SD. The internal validity of discrete choice experiment data: a testing tool for quantitative assessments. Value Health. 2019;22(2):157-60. 